\title{
Research Progress and Prospect of Blockchain + Agricultural Science Data Management
}

\author{
Lina Lu', Shijie Shang ${ }^{1}$ Xiao Yu ${ }^{2 *}$, Lihong Yin1, Jufeng Yin1 \\ ${ }^{1}$ School of Management, Shandong University of Technology, Zibo, China \\ ${ }^{2}$ School of Computer Science and Technology, Shandong University of Technology, Zibo, China \\ Email: think0759@126.com,3050651@qq.com, ^neaufish@163.com, 526708502@qq.com,784275614@qq.com
}

How to cite this paper: Lu, L. N., Shang, $\mathrm{S}$. J., Yu, X., Yin, L. H., \& Yin, J. F. (2021). Research Progress and Prospect of Blockchain + Agricultural Science Data Management. Voice of the Publisher, 7, 163-171. https://doi.org/10.4236/vp.2021.74013

Received: November 25, 2021

Accepted: December 21, 2021

Published: December 24, 2021

Copyright ( 2021 by author(s) and Scientific Research Publishing Inc. This work is licensed under the Creative Commons Attribution International License (CC BY 4.0).

http://creativecommons.org/licenses/by/4.0/ (c) (i) Open Access

\begin{abstract}
Agricultural data management plays an important role in the development of agriculture and even the national economy. Data has been written into the national documents as a factor of production, and its importance is not strict. As the technical support of agricultural data management, blockchain, big data and Internet of Things technology drive the rapid development of agricultural data management. In this paper, the application of blockchain in agricultural data management in the past five years is described in a panoramic manner by using data visualization method. At the same time, content analysis is carried out based on literature content, and the research status at home and abroad is summarized. 92/5000. Through analysis, it is concluded that the application of blockchain technology in agricultural data management mainly includes blockchain + agricultural Internet of Things mode, blockchain + agricultural supply chain mode, blockchain + agricultural data sharing mode, etc. In the future, we need to further expand the application mode of blockchain technology in agricultural data management.
\end{abstract}

\section{Keywords}

Blockchain, Agricultural Data, Data Management

\section{Introduction}

Agricultural data is an important part of data. When we manage agricultural data in the whole life cycle, we use many key technologies, such as big data sharing technology, blockchain technology and cloud storage. Blockchain technology has solved some bottleneck problems in agricultural data protection, data storage and data sharing, and provided impetus for agricultural data sharing. This paper analyzes the status quo of research at home and abroad to find the key application fields and methods and technologies of blockchain in agricultural data 
management.

Blockchain technology is penetrating into agricultural data management with its decentralization, smart contract, consensus mechanism, privacy protection, encryption technology and other methods. Blockchain technology can reduce asymmetric access control of information, and all blockchain participants get the same information. It is a modern P2P technology. Applied to distributed computing and distributed data sharing and storage, we need to seize technological opportunities to accelerate the process of agricultural data management and encourage agricultural organizations to continue to innovate and develop distributed ledger technology solutions. The application of blockchain technology in agricultural data management is mainly in the following areas: agricultural Internet of Things, agricultural supply chain, agricultural data sharing. In this paper, the Chinese database is used as the data source for keyword co-occurrence analysis, to find out the main research hotspots in the field, and find out the research topics of highly cited papers and hot papers, and the application of key technologies in agricultural data management.

\section{Application Research Status at Home and Abroad}

\subsection{Literature Statistics on the Application of Foreign Blockchain in Agricultural Data Management}

Data are obtained from Web of Science, and subject retrieval is conducted using "Blockchain + data" as the keyword. As of July 12, 2020, the data is 2153 pieces of data. As can be seen from the figure, this study started in 2016, and only one related literature was published in 2015. In particular, it has been growing rapidly since 2018. With the expansion of the application field of blockchain technology, not only bitcoin, more and more researchers have paid attention to it and applied it in related fields. At the same time, considerable research results have been obtained in data management. Figure 1 shows the year-by-year statistics of the amount of literature on blockchain data.

\subsection{Research Hotspot Analysis on the Application of Blockchain in Data Management Abroad}

The data source is the data set of web of Science core collection database, the retrieval year is 1986 to present, and the retrieval date is July 2, 2020. The retrieval method is subject, and "Blockchain + Agriculture" is used as the key word. Only 37 literatures are detected. It cannot be analyzed from the panorama, so the search scope is expanded, and 1016 literatures are obtained by using "Blockchain + Data" as the keyword. Through the processing of the search results and adding keywords as the output item, the knowledge graph visualization tool VosViewer1.6.8 is used, and the threshold is set to 5. There are 232 keywords in the software statistical database, and 188 effective keywords are obtained after manual removal of repetitive and meaningless keywords. The network visualization analysis of keywords in the data is carried out, and the visualization panorama 2 is obtained as in Figure 2. 


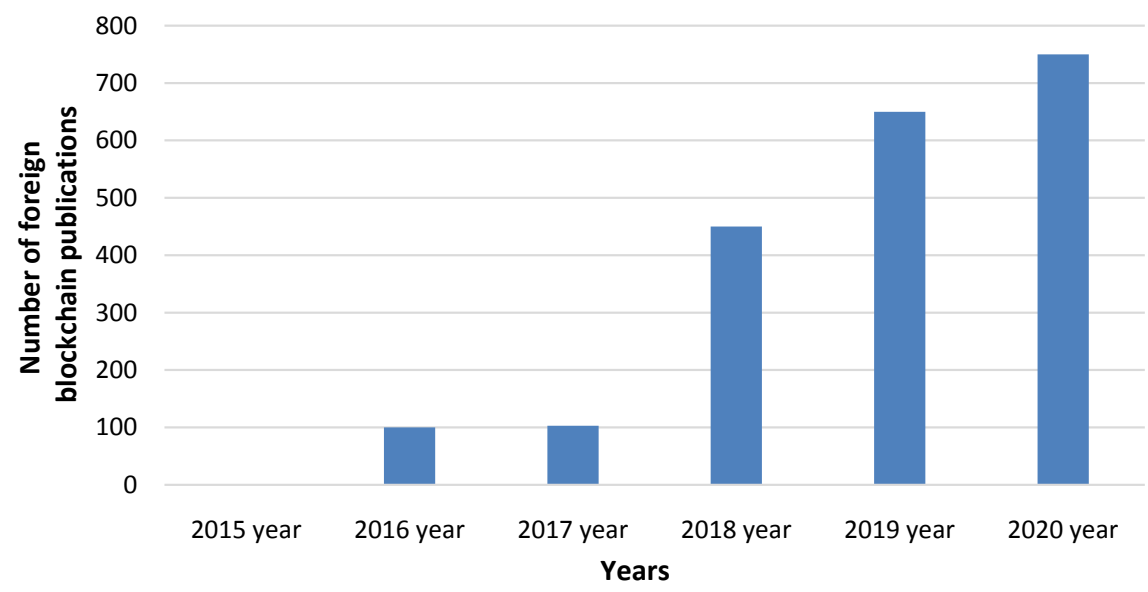

Figure 1. Statistics of foreign blockchain publications in data in recent years.

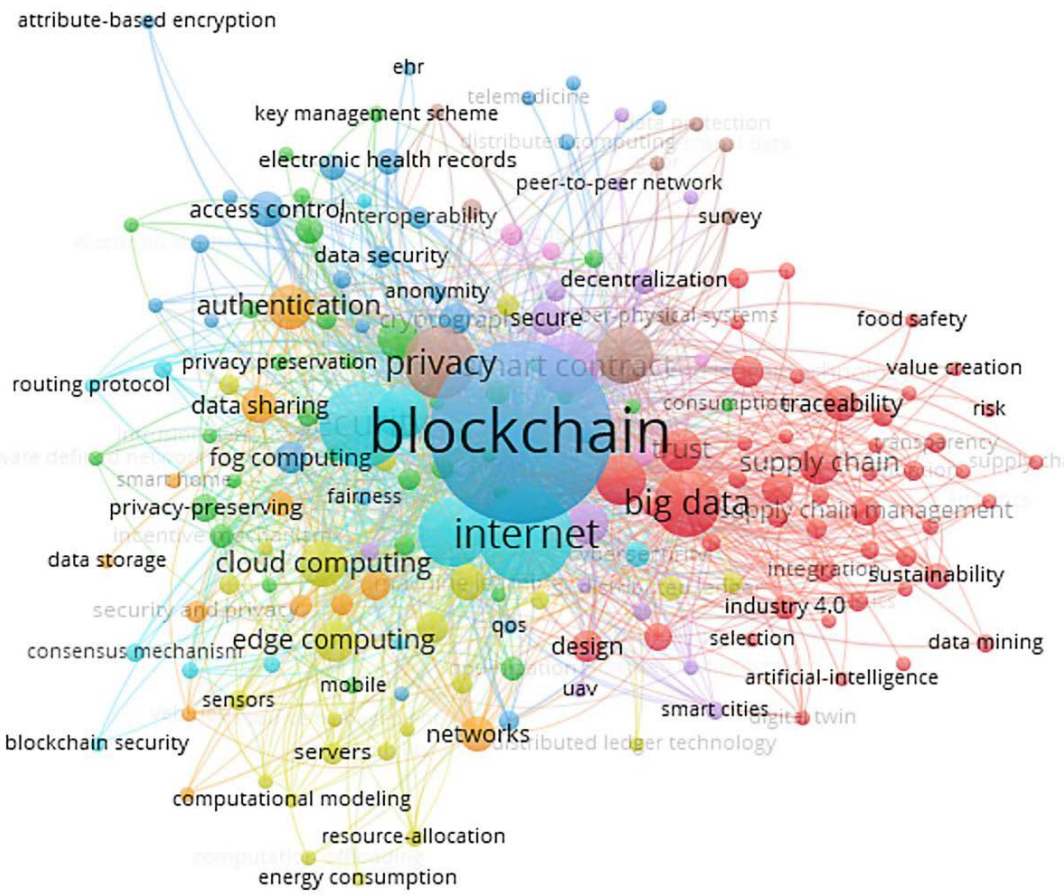

Figure 2. Co-occurrence analysis of foreign keywords "Blockchain + Data".

\subsection{Literature Statistics on the Application of Blockchain in Data Management in China}

The data came from the periodical database of China National Knowledge Network (CNKI), and 1912 articles were obtained by subject retrieval with "blockchain + data" as keywords. The statistics of the number of articles are shown in Figure 3. This figure adopts CNKI's own visual analysis tool for analysis.

\subsection{Research Hotspot Analysis on the Application of Blockchain in Data Management in China}

The data source is the data set of CNKI periodical database, the retrieval year is 1999 to present, and the retrieval date is July 2, 2020. The subject is the retrieval 


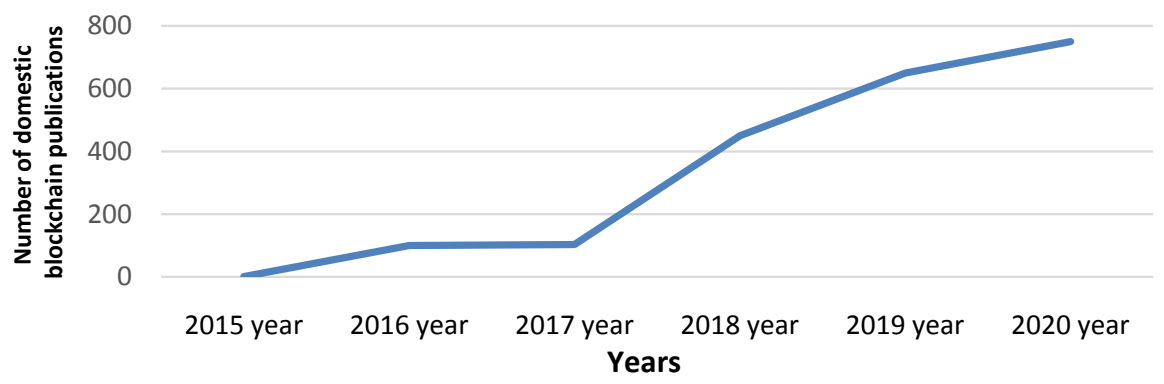

Figure 3. Statistics on the number of domestic blockchain publications in data in recent years.

approach, and "blockchain + data" is used as the key word for retrieval. 3540 literatures are detected, and the output file format is Refwork. Using the knowledge graph visualization tool VosViewer1.6.8, the threshold is set to 5, and the software statistical database contains 515 keywords. After manually removing repetitive and meaningless keywords, 383 effective keywords are obtained. A visual knowledge graph is obtained, from which keywords related to data management are selected by color. To CNKI "were used to blockchain + data sharing" with 217 references, "blockchain + data store" with 204 references, "blockchain + data security" with 274 references, "blockchain + distributed storage" with 107 references, "blockchain + privacy protection" with 231 references, through these five files. The network visualization analysis is carried out on the keywords of the data. The threshold value is 5, 144 keywords are obtained, and 79 English keywords are removed to obtain 65 keywords. Table 1 is the keywords summary of Chinese Atlas.

\subsection{Application Status of Domestic Blockchain in Agriculture}

This paper mainly studies the application of blockchain in agriculture data management, because in order to blockchain + agricultural data management as the theme of the data is very little, not enough to make a visual knowledge map, so this paper expanded the scope, to journals in CNKI database as the data source, with "blockchain + agriculture" as keywords retrieval, got 173 data, research hotspots include agricultural Internet of Things, agricultural products, artificial intelligence, decentralization, smart contracts, big data, smart agriculture, food safety, traceability, agricultural supply chain, rural revitalization, consensus mechanism, agricultural big data, rural finance, etc.

\subsection{Research Review}

Network keyword co-occurrence analysis shows that with the improvement of blockchain technology structure in 2013, the importance of blockchain in the field of agricultural data management has been increasing since 2016. There have been some literature studies on the data management application of blockchain at home and abroad, but there are relatively few literatures on the combination of blockchain with agriculture. Blockchain represents one of the fastest 
Table 1. Keyword word frequency of domestic research on the combination of blockchain and data management.

\begin{tabular}{|c|c|c|}
\hline Keywords & word frequency & word chain \\
\hline Blockchain & 590 & 1806 \\
\hline Protection of privacy & 127 & 576 \\
\hline Blockchain technology & 121 & 216 \\
\hline Data sharing & 103 & 419 \\
\hline Smart contract & 80 & 420 \\
\hline Decentralization & 67 & 281 \\
\hline Data security & 56 & 216 \\
\hline Distributed storage & 54 & 210 \\
\hline Big data & 40 & 147 \\
\hline Bitcoin & 35 & 141 \\
\hline Internet of Things & 34 & 142 \\
\hline Consensus mechanism & 33 & 163 \\
\hline Data store & 31 & 113 \\
\hline Alliance chain & 28 & 115 \\
\hline Artificial intelligence & 16 & 52 \\
\hline Digital currency & 12 & 39 \\
\hline Distributed ledger & 11 & 42 \\
\hline Access control & 9 & 55 \\
\hline Cryptography & 9 & 58 \\
\hline
\end{tabular}

growing emerging technology, it aims at a given data management in agriculture provide transparency of whole life cycle, so as to realize the traceability of the data is simple and safe, it is a kind of added value for data management in agriculture, along with the network threat systematic evolution of emerging technology and complexity, Empowering blockchain helps achieve the important goal of combating cyber security risks to fully protect the integrity of agricultural data.

\section{Blockchain Simulation}

Although some studies on the application of blockchain in data management have appeared in the literature, there is very little if it is applied in the field of agricultural data management, and even less about the application and simulation. However, more and more scholars have begun to pay attention to the application of blockchain, the management of agricultural supply chain, the construction of agricultural Internet of Things and the management of agricultural data. After the panoramic analysis of the above content, we find out some simulation and application cases of blockchain from important literature in the reading database, mainly focusing on the following aspects. 


\subsection{Blockchain + Agricultural Supply Chain Model}

Blockchain technology promises to improve transparency and accountability in supply chain networks, thereby making value chains more flexible. In particular, blockchain-based applications have the potential to generate breakthroughs in three areas of supply chain: visibility, optimization, and demand (Wang, 2020) Agricultural supply chain is responsible for the complex system of agricultural products in the market circulation, in the context of precision agriculture, information and communication technologies (for example, farm through smartphone application of remote control, remote monitoring farm equipment, etc.) can be further in the blockchain infrastructure implementation, make the new farm system and electronic agricultural planning become possible. Allow engineering safety and trust in sustainable agricultural development and transparent data collection to immutable and decentralized future management (Xia, 2020).

Foreign research. First, the attribute of blockchain traceability. Most of the "Blockchain + Agriculture" in Chinese and foreign languages in this paper is about agricultural supply chain, and the focus of agricultural supply chain application is "agricultural product traceability in agricultural supply chain". After the application of blockchain in agricultural supply chain, all transactions are stored in the supply chain in a reliable and immutable manner and recorded in the supply chain in a highly transparent manner (Casino et al., 2019). K. Salah et al used Ethernet and smart contracts to track and execute business transactions, eliminating middlemen and processing central points, thus achieving soybean traceability across agricultural supply chains (Salah, 2019). In the supply chain application environment, the blockchain ledger will record the products from suppliers to consumers in detail. Through the use of RFID technology and GPS technology, the goods will be tracked at each stage. Trust will be guaranteed through the immutability of technology, because fraud can be easily detected in any part of the supply chain.

Domestic research. In terms of literature, the CNKI database was searched for all types of literature, and 241 pieces of data were obtained by "blockchain + agricultural supply chain". Among them, 116 are about supply chain traceability. First, traceability of agricultural products. Yu Hemong et al. adopted the node authorization method, which took into account the two aspects of privacy data encryption protection and supply chain traceability data public supervision, and solved the data sharing problem among multi-nodes of rice supply chain production, processing and circulation (Francesca et al., 2019). Ren Shougang et al. designed an information traceability platform for the whole industry chain of crops based on CSBFT blockchain. Compared with the existing food traceability platform based on blockchain, CSBFT algorithm has higher security and smaller delay (Salah et al., 2019). Agricultural supply chain finance. The application of blockchain in agricultural supply chain finance is mainly in the prevention of credit risks. Xia Meiyue and Zhao Huawei analyzed the causes and effects of credit risks in agricultural supply chain finance, and explored the use of blockchain 
technology advantages to improve the credit mechanism of agricultural supply chain (Wang et al., 2019). Yin Yanfei and Wu Biti put forward the application prospect and application mode based on digital finance in supply chain (Li et al., 2019). Third, trust in transactions. MAO Dianhui et al. proposed a food trading mechanism based on alliance chain and developed a safe food trading system using consortium blockchain FTSCON to solve the trust problem in food trade (Yang et al., 2019).

\subsection{Blockchain + Agricultural IoT Model}

The Internet of Things presents a number of technical challenges: cybersecurity, privacy, massive data management, lack of standardization and interoperability, and the complexity and heterogeneity of the technologies involved in the Internet of Things require specific skills in designing, implementing, and deploying solutions. With blockchain de-centralization networking, in recognition of the opportunities blockchain offers, researchers and developers are starting to create decentralized applications for the Internet of Things, with network edges gaining more functionality than the cloud, such as fog and Mist frameworks. The integration of blockchain and Internet of Things has brought new ideas to the development of agricultural data management. A number of solutions for Internet of Things data management based on blockchain have been proposed, providing guaranteed integrity and flexibility for data management.

\subsection{Blockchain + Agricultural Data Sharing Model}

Blockchain + agricultural data sharing is inseparable from agricultural supply chain and agricultural Internet of Things research, and many cases are integrated. The application of blockchain in agricultural data sharing is mainly reflected in distributed storage of agricultural data, cryptography, edge computing, agricultural data security and other aspects.

Foreign research. Lei Hang et al. proposed a data integrity platform based on blockchain secure fish farm, which aims to provide safe storage for fish farm users to preserve a large amount of agricultural data that cannot be tampered with. Meanwhile, they proposed the application of distributed classification technology of agricultural data storage to ensure the integrity of agricultural data (Ali et al., 2019). Francesca Antonucci has introduced an ontology-based blockchain modeling method that combines Internet of Things devices for data capture and supply chain source data sharing (Francesca et al., 2019).

Domestic research. In terms of data sharing, such as noctis mainly based on blockchain network are studied to establish a set of data security in the chain of share system, access control algorithm based on the properties of the encryption cipher strategy and homomorphic encryption algorithm provides reliable chain data sharing, data sharing framework put forward chain to protect the privacy of user data security (Zhang \& Hu, 2020); Yu Jingang et al. proposed a blockchain-based data sharing model for the Internet of Things by taking advantage 
of the fact that blockchain can solve trust problems in a decentralized manner (She et al., 2020). In terms of agriculture data sharing, document types are mainly concentrated in the patent literature, patent literature is focused on the precision, such as: one based on blockchain agricultural data sharing method, a kind of agricultural data sharing system based on blockchain, a method based on blockchain of agriculture data acquisition and sharing system, a kind of agricultural data sharing system and the method based on blockchain, etc.

\section{Conclusion and Prospects}

This paper makes a visual analysis of the application of blockchain in agricultural data management in recent years, especially in the past five years, based on the analysis of the current situation and hotspots of the application of blockchain data management at home and abroad. This paper interprets and reviews the key literature and hot literature in the field; the article still needs further research in theory and application, and needs more in-depth case analysis and future trend analysis.

Blockchain has great potential in agricultural data management. In order to better integrate blockchain technology into the application of agricultural data management, the author puts forward some suggestions as follows: Not all agricultural data management can apply blockchain technology. We need to conduct in-depth analysis on the application scenarios of blockchain, expand the application scenarios of blockchain agricultural data management, conduct in-depth mining in agricultural data management, and find the key points of integration of the two. Secondly, in the theoretical aspect of the application of agricultural data management of blockchain, the corresponding theoretical research is formed, and the construction of the application framework of agricultural data management of blockchain is a development direction in the future. According to the existing research results, theoretical research is formed to guide the application of agricultural data management of blockchain in the future. Thirdly, in terms of technology, it is still necessary to integrate blockchain with agricultural big data technology and agricultural Internet of things technology, and the level of agricultural data acquisition, data quality control, database construction and data mining application is low. The information foundation of agricultural network is weak, so we should give priority to strengthening the construction of network information infrastructure, including the construction of Internet of things and big data system, and lay a good foundation for the application of blockchain (Lei et al., 2020). Finally, there are still some challenging research areas in the application of blockchain in agricultural data management, such as the integration of blockchain and agricultural Internet of Things technology, ownership issues of blockchain technology, scalability analysis of blockchain-based solutions, etc., all of which need further research in the near future (Liu et al., 2020).

\section{Foundation Item}

National Social Science Foundation of China (18BTQ062) Research on the con- 
struction of agricultural science data Supervision mechanism under big data environment.

\section{Conflicts of Interest}

The authors declare no conflicts of interest regarding the publication of this paper.

\section{References}

Ali, M. S. et al. (2019). Applications of Blockchains in the Internet of Things: A Comprehensive Survey. IEEE Communications Surveys \& Tutorials, 21, 1676-1717. https://doi.org/10.1109/COMST.2018.2886932

Casino, F. et al. (2019). A Systematic Literature Review of Blockchain-Based Applications: Current Status, Classification and Open Issues. Telematics and Informatics, 36, 55-81. https://doi.org/10.1016/j.tele.2018.11.006

Francesca, A. et al. (2019). A Review on Blockchain Applications in the Agri-Food Sector. Journal of the Science of Food and Agriculture, 99, 6129-6138. https://doi.org/10.1002/jsfa.9912

Lei, H., Ullah, I., \& Kim, D. H. (2020). A Secure Fish Farm Platform Based on Blockchain for Agriculture Data Integrity. Computers and Electronics in Agriculture, 170, Article ID: 105251. https://doi.org/10.1016/j.compag.2020.105251

Li, M. J. et al. (2019). Design of Food Safety Traceability System Based on Block Chain. Food Science, 40, 279-285.

Liu, Y. S. et al. (2020). Sharing Data Security System Based on Block Chain Research. Journal of Big Data, 6, 92-105. https://kns.cnki.net/kcms/detail/10.1321.G2.20200622.1046.002.html

Salah, K. et al. (2019). Blockchain-Based Soybean Traceability in Agricultural Supply Chain. IEEE Access, 7, 73295-73305. https://doi.org/10.1109/ACCESS.2019.2918000

She, W. et al. (2020). Location Privacy Protection Model of IoT Nodes Based on Block Chain. Journal of Applied Science, 38, 139-151.

Wang, F. (2020). Ten Problems on Market Allocation of Data Elements. Books and Information, 3, 9-13.

Wang, J. et al. (2019). Application of Computer in China. Computer Applications, 39, 2438-2443.

Xia, Y. K. (2020). Market-Oriented Allocation of Data Elements and Deepening the Reform of Government Data Governance Mode. Books and Information, 3, 14-16.

Yang, X. T. et al. (2019). Information Storage Model and Query Method of Agricultural Products Traceability System Based on Block Chain. Transactions of the Chinese Society of Agricultural Engineering, 35,323-330.

Zhang, J. G., \& Hu, X. H. (2020). An Improved Iot Device Based on Ethernet Fang Access Control Study. Computer Engineering, 47, 32-39. 